\title{
Application of routing protocols: Monitoring of Criminal Activities
}

\author{
Shivali Dhaka ${ }^{1}$
}

\begin{abstract}
The world is growing so fast and one of main pillar of this growth is the technology and inside the technology we will found one sub-pillar of its which is generally called "Network". Exchange of routing information between routers and network elements of Local Domain network is possible by instigating Routing Protocols. Different types of crimes that remain unidentified and unknown due to lack of communication between domains and an information system are of huge importance today. So the need is to build a Network which will fit to today's world challenges which we are facing daily. The more the crime rates boost the more security forces have to be armed with a precisely good and well-structured network system that can help them to track down criminal information and keep a judiciary record.This project focuses on centralizing the security forces information and data with help of routing protocols OSPG and EIGRP, which can be useful for tracking crimes and criminal in a local area.
\end{abstract}

Keywords-DynamicRoutingProtocol, OSPF, EIGRP, FTP, SMTP, HTTP and DNS.

\section{INTRODUCTION}

Since 1980, Network is using a Dynamic routing protocol that describes the capability of a system, through which routes are considered by their destination, to alter the path that the route takes through the system in response to a change in conditions.Dynamically Network destinations are discovered with use of these protocols and thus have a vast impact on utilization of network resources. Dynamic routing protocol is also named as adaptive routing as it allows as many routes as possible to remain valid in response to the change. AsNetwork is expanding and becoming more complex, newrouting protocols haveemerged. Network Topology is based on function of router to receive data packets and forwarding to next hops in the inter-network while maintaining routing table. Routing table encloses information regarding network source and destination hops and other network elements. The packet will only be recognized by destination only if its information is stored in routing table, otherwise packet will be abandon. Routing protocols help in maintaining routing information in routers according to their own algorithms. Routing protocols are categorized into: Static and Dynamic. Static routing happens when an administrator manually assigns the path from source to destination network. It provides more security to network. The main drawback of static routing is that when a link fail in the internetwork the entire network goes down. This is feasible in Small networks but not in the large networks. Dynamic routing is the process in which routing tables are automatically updates by routing protocols and all the processes are done dynamically through the dynamic routing protocols. Asshown in Figure 1,dynamicroutingprotocolsareoftwomaintypes, whichareinteriororexterior. Interiorprotocolsarethosethatoperate withinonesameautonomoussystem(AS)androutepacketsbetweendifferent AS and thereshouldbeanexteriorprotocol configured.Interiorroutingprotocolsarealso classified intwoclassesnamelydistance vector andlink state.

A.Distance vector protocols include-

- RoutingInformationProtocol(RIPversion1andversion2)

- InteriorGatewayroutingprotocol(IGRP)

- EnhancedInteriorGatewayroutingprotocol(EIGRP)

\section{B. Link state routing protocols include-}

- OpenShortestPathFirst(OSPF)

- IntermediateSystemtoIntermediate System(IS-IS)

\footnotetext{
${ }^{1}$ Department of Computer Science Amity University, Gurgaon, Haryana, India.
} 


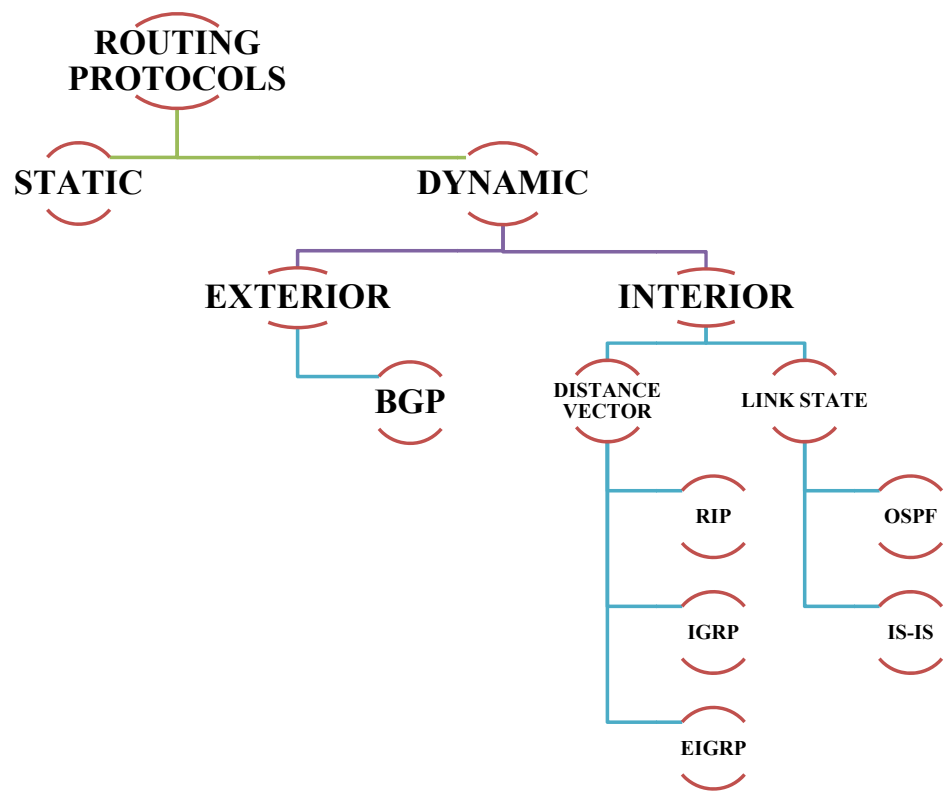

Figure 1. Classification of Routing protocols

Exterior routing protocol comprises of Border Gateway Protocol (BGP).Inlinkstaterouting,everynodehas in its routing tablearoadmapofconnectivity tothenetwork,showingwhichnodeare connectedtowhich othernodes in the network. Every possible node in the network does calculation of best logical path that forms the contents ofroutingtable.InLink state routing protocol theonly communicationshared between nodes isconnectivityrelated. The rest of the paper is organized as follows. Route redistributionare explained in section II. Design and implementations are presented in section III. Concluding remarks are given in section IV.

\section{ROUTE REDISTRIBUTION}

\section{A. Introduction}

For simplicity and ease of management, it is preferable to employ a single routing protocol in an internetwork environment rather than multiprotocol. Unfortunately, this is not always possible, making multi-protocol environments common. Route Redistribution allows routes from one routing protocol to be advertised into another routing protocol. The routing protocol receiving these redistributed routes usually marks the routes as external. External routes are usually less preferred than locally-originated routes. At least one redistribution point needs to exist between the two routing domains. This device will actually run both routing protocols. Thus, to perform redistribution in the following example, Router B would require at least one interface in both the EIGRP and the OSPF routing domains:

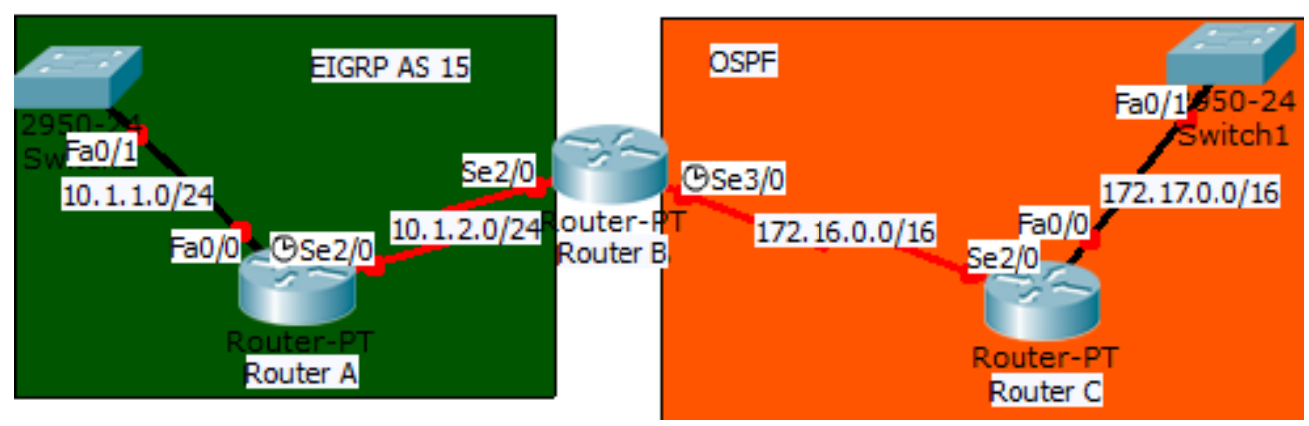

Figure 2. Shows as an example of two autonomous systems using two different routing protocols A, uses EIGRP and C, uses OSPF and both share their route through $\mathrm{B}$, whose redistributing the routes. 


\section{B. MODE OF CONFIGURATION}

- Redistributing into EIGRP

EIGRP is a hybrid routing protocol that, by default, uses a composite of bandwidth and delay as its distance metric. EIGRP can additionally consider Reliability, Load, and MTU for its metric.To redistribute all OSPF routes into EIGRP:

RouterB(config)\# router eigrp 15

RouterB(config-router)\# network 10.1.2.0 0.0.0.255

RouterB(config-router)\# redistribute ospf 20 metric 10000100025511500

First, the router eigrp process was enabled for Autonomous System 15. Next, EIGRP was configured to advertise the network of 10.1.2.0/24. Finally, EIGRP was configured to redistribute all ospf routes from process ID 20, and apply a metric of 10000 (bandwidth), 1000 (delay), 255 (reliability), 1 (load), and 1500 (MTU) to the redistributed routes.

EIGRP, by default, will auto-summarize internal routes unless the no auto summary command is used. However, EIGRP will not auto-summarize external routes unless a connected or internal EIGRP route exists in the routing table from the same major network of

RouterB(config)\# router ospf 20

RouterB(config-router)\# network 172.16.0.0 0.0.255.255 area 0

RouterB(config-router)\# redistribute eigrp 15

RouterB(config-router)\# default-metric 30

First, the router OSPF process was enabled with a process-ID of 20. Next, OSPF was configured to place any interfaces in the network of 172.16.0.0/16 into area 0. Then, OSPF will redistribute all eigrp routes from AS 15. Finally, a default-metric of 30 was applied to all redistributed routes.

\section{DESIGN AND IMPLEMENTATION}

\section{A. Introduction}

The Security forces is composed bodies of people endowed by the state to enforce the law protect property and limit civil disorder. Their powers include the legitimized use of force. The term is most commonly associated with law enforcement agencies of sovereign state that are authorized to exercise the law enforcement agencies power of that state within a defined legal or territorial area of responsibility. Police forces are often defined as being separate from Military or other organizations involved in the defense of the state against foreign aggressors; however, Gendarmerie is military units charged with civil policing. The Indian central government maintains several security police forces:

CISF is The Central Industrial Security Force (established in its present form: June 15, 1983) is a paramilitary security force in India. To protect public properties and private properties following are the CISF duties:

- CISF to protect public sector.

- CISF to protect private sector.

- Airport Security

It is one of the largest central paramilitary forces in India. Strength is nearly 105,000 and rivals other countries when it comes to a Government agency providing security to such a large number of industries. Many of the international airports in India were the responsibility of the city or district police.Railway Protection Force (RPF) known for protecting the railways of India and ensuring safety of citizens in trains. The Defense Security Force protects Ministry of Defense property.

\section{B. Requirement}

Making a special network for such department will be of a huge importance, this can help them to:

- Centralization of the police data and information

- Protect the population and private properties

- Manage the migrations and refugees movements.

- Maintain a central record of all the Judiciary cases at each level.

- Exchange emails whenever required.

- Exchange ideas and strategies of dealing with criminals.

- Have a local record of all the cases and all the prison effectives. 
- Know who is In and who is Out of the prison.

- Know who is coming in and going out of the Locality.

- Know the sentence of each detained.

\section{Cost}

Building such network will be costly because it requires good quality network devices(Routers, Servers, Switches and computes) apart of that it requires a dynamic website and a constant maintenance to avoid failures. High security should be to the program to prevent loss of data and different cyber attacks and crimes.

\section{Network topology}

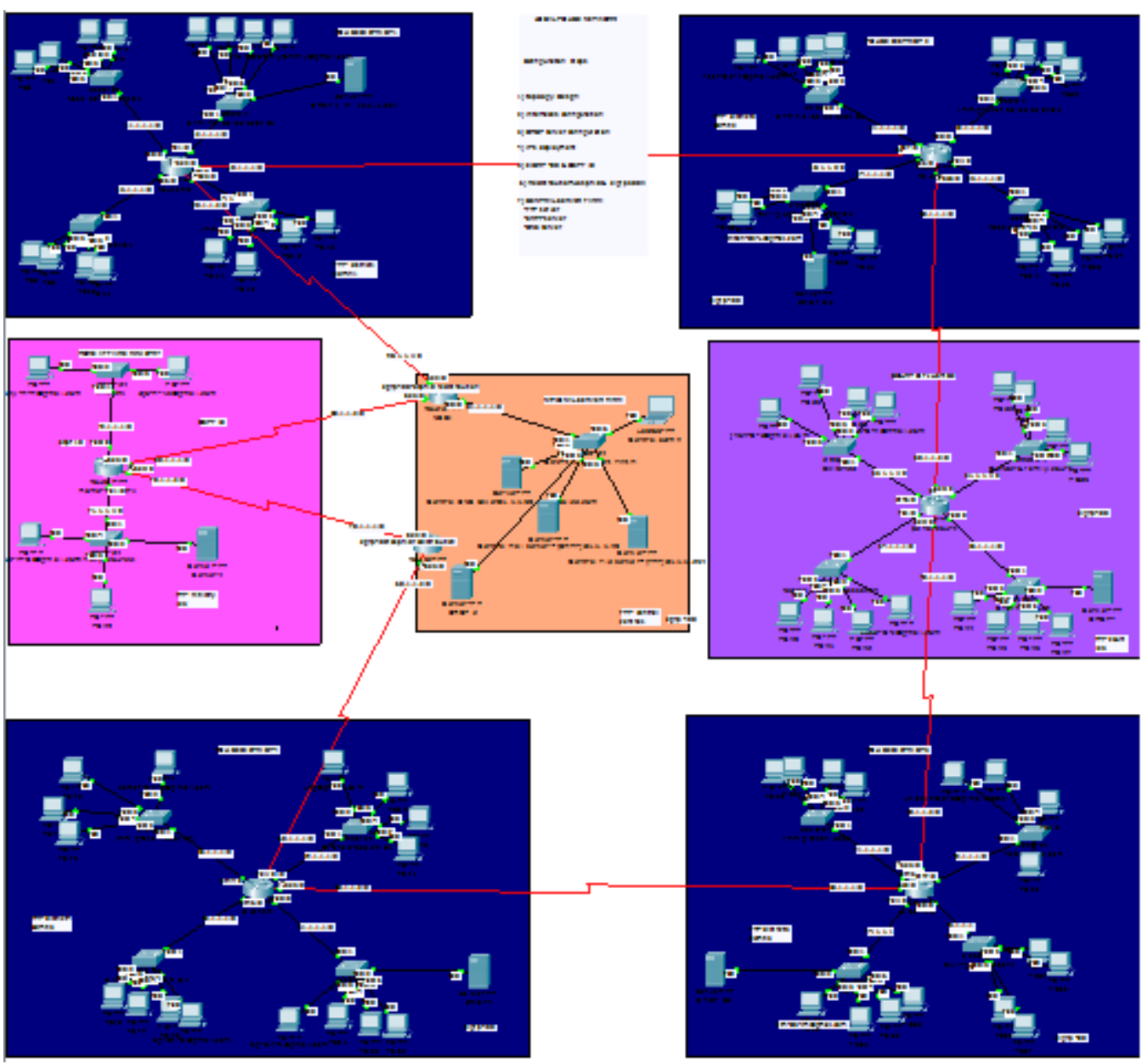

Figure 3. Local Security Forces network topology

\section{E.Monitoring of crimes}

Topology-

The Law enforcement agencies network is located in a Province that is having four districts and in each district we are having one a police body that is having also four police sub-departments:

- Immigration police department

- $\quad$ Road police department

- Criminal police department

- Guard civil police department

All the four police department are geographically located on different sites but there are one Court of justice, wherever they are having a judiciary case, they will after $24 \mathrm{~h}$ transfer it to the justice and the last will judge and give a sentence.

The court of justice is having fourdepartments too which are:

- Federal court of cassation 
- Gender and family court

- Criminal court

- Lawyers and Judges cabinet

So in common the two bodies are dependent of the Home affairs minister cabinet they are working in coordination and all the big decision are taken by the minister cabinet and expend in all the departments. The network project is having a central server farm, where all the data and information are kept.

Design -

- The blue areas are the four Police departments.

- The purple area is the Court of justice.

- The violet area is the Home ministry.

- The orange area is the Central server farm.

- Devices used:

* 1 Router/police district, 4 Switches/department and 1 DHCP server

* The Server Farm is having 3 main servers

* FTP server (it contains all the files transferred from one site to another)

* DNS server (it having the domain names of the police website)

- HTTP server (this server is helping the polices department to access the web site and the network administrator to upload everyday new records)

* SMTP server (this server is used form mail transfer in the entire network)

\section{Configuration steps-}

Step 1: Topology design

Step 2: Network interfaces configuration

Step 3: DHCP server configuration for IP's deployment overall the network dynamically

Step 4: Routing protocols configuration EIGRP 400 and OSPF 20

Step 5: Redistribution configuration between the two protocols

Step 6: Central server farm configuration

- FTP Server

- SMTP Server

- DNS Server

- HTTP Server

\section{CONCLUSION AND FUTURE SCOPE}

The project concludes the projection of making a secure world and to enforce with this idea because the world need to be secure, the only one way we can be secure is by providing our Law Enforcement agencies the good infrastructure that can help them to work and cooperate between them even if they are far geographically. A good network that is well maintained and secure is of a great importance.

For the future expectation the projects explores to add more security features like:

- SNMP server for more security

- Firewall

- SYSLOG and NTP server

- NAT and port security

The further work of this project doesn't excludes anyone, this is open to anybody who would like to expend this idea at the wide area like at a state level or a country level, so that different states will have their own center which will be the sub-data center and all the data center will be stored at a central farm which can help in keeping record of not only the crimes and criminals but also for all the citizen of the country.

\section{REFERENCES}

[1] Understandingtheprotocols underlying dynamicRouting, CNET Networks,andRetrieved2008-10-17. 
[2] Rick GrazianiandAllanJonson, 2008, RoutingProtocols and Concepts,CCNAexploration companion guide,PearsonEducation.

[3] C C N P 1 AdvancedRouting CompanionGuide,Indianapolis:CISCO Press 2004,pp.93f,ISBN 1-58713-135-8.

[4] CISCOOSPFDesignGuide,http://www.cisco.com.

[5] Networking ProtocolConfigurations,CISCO systems retrieved 2008-10-16.

[6] DejanSpasov,MarjanGushev,“OntheConvergence of Distance VectorRouting Protocols”,ICT2012.

[7 Exposito"Easy-EIGRP:ADidacticApplicationfor Teaching andLearning of theEnhancedInteriorGatewayRouting Protocol'ICNS'10 Proceedings of the SixthInternationalConference onNetworkingand Services, 2010.

[8] PankajRakheja,Prabhjotkaur, Performance Analysisof RIP,OSPF, IGRPandEIGRPRoutingProtocolsina Network, International Journal of Computer Applications, 2012. Savage,Slice "Enhanced Interior Gateway Routing Protocol'InternetEngineering Task Force, 2013.

[9] Muhammad TayyabAshraf," "How to Select a Best Routing Protocol for your Network" Canadian Journal on Network and InformationSecurityVol. 1,No.6, August2010.

[10] AnkitSharma, Sheilly Padda,“Configuringan EIGRPbased RoutingModel”,InternationalJournalofScientificandResearch Publications, 2012.

[11] AlexHinds,AnthonyAtojoko,ShaoYingZhu.(2013).EvaluationofOSPFandEIGRPRoutingProtocolsfor IPv6.InternationalJournalofFutureComputer andCommunication, 2(4).

[12] Anibrika Bright SelormKodzo, GolapKanti Dey, Md.MobasherAhmed, KaziTanvirAhmmed.(2015)Performance analysis and redistribution among RIPv2, EIGRP \& OSPFRouting Protocol. https://www.researchgate.net/publication/283015133.

[13] Forouzan, B.A.(2010).TCP IP ProtocolSuite(4ed.). NEWDELHI, INDIA:McGrawHillEducation.

[14] Haresh N.Patel, Prof.Rashmi Pandey.(2014). Extensive ReviewsofOSPFandEIGRPRoutingProtocols based on RouteSummarization andRouteRedistribution.InternationalJournalofEngineeringResearchand Applications, $4(9($ version 4$))$. 